\title{
Caracterización clínica y epidemiológica de los casos de dengue: experiencia del Hospital General de Culiacán, Sinaloa, México
}

\author{
María Guadalupe Ramírez-Zepeda, ${ }^{1}$ Héctor Eduardo Velasco-Mondragón, ${ }^{2}$ \\ Celso Ramos, ${ }^{3}$ Javier E. Peñuelas, ${ }^{4}$ Marco Antonio Maradiaga-Ceceña, ${ }^{5}$ \\ Joel Murillo-Llanes, ${ }^{5}$ Ramón Rivas-Llamas ${ }^{5}$ y Ricardo Chaín-Castro ${ }^{5}$
}

Forma de citar

Ramírez-Zepeda MG, Velasco-Mondragón HE, Ramos C, Peñuelas JE, Maradiaga-Ceceña MA, Murillo-Llanes, J, et al. Caracterización clínica y epidemiológica de los casos de dengue: experiencia del Hospital General de Culiacán, Sinaloa, México. Rev Panam Salud Publica. 2009;25(1):16-23.

RESUMEN Objetivo. Documentar las características clínicas y epidemiológicas de los pacientes con dengue atendidos en un hospital de Culiacán, Sinaloa, México.

Métodos. Estudio transversal, observacional y analítico realizado en el Hospital General Dr. Bernardo J. Gastélum de Culiacán entre el 1 de octubre y el 2 de diciembre de 2003. Se evaluó la asociación entre las variables independientes (las características clínicas y epidemiológicas de los pacientes) y la variable dependiente (diagnóstico confirmado de dengue hemorrágico) mediante el análisis de regresión simple. Las variables que mostraron una relación significativa $(\mathrm{P}<0,05)$ se incluyeron en el análisis de regresión logística multifactorial.

Resultados. En los 241 casos que cumplieron los criterios de inclusión para este estudio (207 de dengue y 34 de dengue hemorrágico), la edad promedio fue de 34,7 $\pm 15,1$ años. Según los resultados del análisis multifactorial ajustado por la edad, el sexo y la presencia de casos de dengue en la localidad, las variables con valor predictivo de una mayor gravedad de la enfermedad fueron: la presencia de ascitis (OR =22,12; IC95\%: 5,00 a 97,87), la gingivorragia $(O R=7,35$; IC95\%: 2,11 a 25,61), la hematemesis (OR = 7,40; IC95\%: 1,04 a 52,42), la trombocitopenia (plaquetas entre $40001 / \mathrm{mm}^{3}$ y $\left.60000 / \mathrm{mm}^{3}\right)(O R=5,43$; IC95\%: 1,58 a 18,72), la hiperemia conjuntival (OR =4,27; IC95\%: 1,37 a 13,28), los vómitos persistentes (OR =3,04; IC95\%: 1,05 a 8,80) y la ausencia de congestión nasal (OR =0,015; IC95\%: 0,0004 a 0,473).

Conclusiones. Se confirmó el valor de la presencia de ascitis, gingivorragia, hematemesis, trombocitopenia (con valores de plaquetas entre $40001 / \mathrm{mm}^{3}$ y $60000 / \mathrm{mm}^{3}$ ) y vómitos persistentes como signos de alarma que anuncian la inminencia del choque por dengue. Se observaron conteos plaquetarios $>100000 / \mathrm{mm}^{3}$ en casos con cuadros clínicos graves (fuga capilar) que no se clasificaron como dengue hemorrágico por no cumplir todos los criterios establecidos por la OMS.

Palabras clave Dengue, fiebre dengue hemorrágica, signos y síntomas, epidemiología, México.

Dirección de Investigación en Políticas para la Salud, Secretaría de Salud, y Facultad de Medicina, Universidad Autónoma de Sinaloa, Sinaloa, México. La correspondencia se debe enviar a María Guadalupe Ramírez Zepeda, Dirección de Investigación en Políticas de Salud, Secretaría de
Salud de Sinaloa, Av. Lázaro Cárdenas y Calle Escuadrón 201, Col. Almada, Culiacán, Sinaloa 80129, Mexico. Correo electrónico: graze75@uas. uasnet.mx

2 Morgan State University, Maryland, Estados Unidos de América.
Instituto Nacional de Salud Pública, Morelos, México.

4 Dirección de Investigación en Políticas de Salud, Sinaloa, México.

5 Hospital General de Culiacán Dr. Bernardo J. Gastélum, Secretaria de Salud, Culiacán, Sinaloa, México. 
El dengue constituye un importante problema de salud pública en todo el mundo, principalmente en las regiones tropicales y subtropicales. Los cuatro serotipos descritos del virus del dengue producen por lo general una enfermedad febril autolimitada. Sin embargo, tiene un espectro clínico muy amplio que va desde formas asintomáticas hasta su forma más grave, el dengue hemorrágico. Este último se caracteriza por la fuga capilar en diversas cavidades, hemorragias, hemoconcentración y trombocitopenia y puede causar la muerte (1).

El dengue es una de las enfermedades infecciosas reemergentes más importantes en el mundo actual (2) y provoca entre 50 y 100 millones de casos anuales en más de 100 países. De esos casos, más de 500000 son de dengue hemorrágico, que además de requerir cuidados intensivos hospitalarios causa alrededor de 24000 defunciones, la mayor parte de ellas en niños $(1,3)$. Durante las epidemias, la tasa de ataque del dengue puede llegar a afectar hasta a $90 \%$ de las personas susceptibles (4), con un índice de letalidad de hasta $5 \%(5,6)$.

La dinámica de transmisión del dengue está estrechamente asociada con la expansión geográfica del virus y de su principal agente transmisor, el mosquito Aedes aegypti. Recientemente se ha demostrado la asociación entre el cambio climático y el incremento de las epidemias a partir del análisis de mapas climáticos y de mediciones de la densidad y la distribución de los principales vectores en países como Colombia, Haití, Honduras, Indonesia, Tailandia y Vietnam (7). Asimismo, algunos estudios han demostrado que la sequía también puede ser un factor importante en la aparición de epidemias, ya que el almacenamiento de agua en los hogares favorece la cría del mosquito vector de la enfermedad $(8,9)$.

Otros elementos importantes que se deben tomar en cuenta para poder establecer medidas eficaces y oportunas de control son el patrón epidemiológico de circulación viral - ya que pueden circular cepas de mayor virulencia o más de un serotipo simultáneamente-, el aumento del número de casos de dengue hemorrágico en zonas endémicas y la detección de casos en nuevas áreas (10-12).

$\mathrm{Si}$ las condiciones ambientales, como la humedad y la temperatura, se mantienen en niveles que favorecen la diseminación de la infección y la población au- menta en la forma proyectada, se prevé que en el año 2055 aproximadamente 3200 millones de personas (34\% de la población mundial) estarán en riesgo de contraer dengue, lo que representará no solamente una enorme carga de enfermedad, sino también una pesada carga para la economía y los sistemas de salud (13).

Entre 2001 y 2005, más de 30 países de las Américas notificaron en conjunto casi 3 millones de casos de dengue, de ellos 65235 de dengue hemorrágico (14). En 2007, la Organización Panamericana de la Salud (OPS) recibió informes de 900000 casos de dengue, 26412 casos de dengue hemorrágico y 317 defunciones en la Región (15).

En México, la primera epidemia de dengue hemorrágico ocurrió en 1995 cuando se registraron 539 personas enfermas (16). A partir de entonces, el número de casos de dengue ha aumentado $\mathrm{y}$, según datos oficiales, la incidencia pasó de 5220 casos en 2003 a 40559 en 2007, con 1776 y 7897 casos de dengue hemorrágico, respectivamente (15). Aunque en la actualidad en México circulan los cuatros serotipos virales, el número de muertes por dengue se ha mantenido por debajo de $1 \%$ del total de casos de dengue hemorrágico (17).

En Sinaloa, uno de los estados federativos del noroeste de México que cuenta con una población de más de 2,6 millones de habitantes, los primeros casos de dengue se detectaron en la década de 1980 y para el año 2002 se habían identificado los serotipos virales 1, 2 y 4 . En ese período se presentaron dos importantes brotes: el primero en 1992 con 5784 casos de dengue, aunque sin casos diagnosticados de dengue hemorrágico, y el segundo en 1995 con 11053 casos de dengue y 161 de dengue hemorrágico. En la temporada invernal de principios de 2003 se informaron más casos de dengue y se detectó por primera vez el serotipo 3. En 1995, la Secretaría de Salud de Sinaloa informó 24818 casos probables de dengue -1241 de ellos confirmados-, 594 de dengue hemorrágico y 7 defunciones (18). Ese año, la incidencia de dengue en el Estado aumentó en los meses de septiembre y octubre y los servicios de urgencias quedaron saturados por los casos sospechados de dengue.

El objetivo del presente trabajo es documentar las características clínicas y epidemiológicas de los pacientes con dengue atendidos en un hospital de $\mathrm{Cu}$ liacán, Sinaloa, México.

\section{MATERIALES Y MÉTODOS}

Se conformó un equipo multidisciplinario integrado por médicos, epidemiólogos, investigadores y personal de enfermería del Hospital General Dr. Bernardo J. Gastélum de Culiacán, capital del estado de Sinaloa, México, con el objetivo de unificar los criterios de diagnóstico clínico y la conducta a seguir durante el tratamiento en esa institución. Como primer paso, ese equipo revisó la guía metodológica propuesta por el Sistema Nacional de Salud, a través del Sistema de Vigilancia de Dengue, para el estudio clínico y epidemiológico del dengue y el dengue hemorrágico (19) y concluyó que no es adecuada, ya que no permite determinar de forma oportuna si se trata de un cuadro de dengue o de dengue hemorrágico. Además de utilizar conceptos que no se corresponden con la clasificación adoptada por la Organización Mundial de la Salud (OMS) — tales como dengue clásico, dengue clásico con manifestaciones hemorrágicas, dengue hemorrágico y síndrome de choque por dengue-- esta guía está compuesta por más de 100 variables, lo que hace muy difícil su aplicación. Ante esta situación se procedió a elaborar una guía de encuesta para el estudio clínico-epidemiológico de los casos de dengue.

Para llegar a un conceso se realizó un estudio transversal, observacional y analítico entre el 1 de octubre y el 2 de diciembre de 2003, período en el que habitualmente se presenta el mayor número de casos de dengue en México, según datos de la Dirección General de Epidemiología de la Secretaría de Salud (19).

Se estudiaron todos los casos con diagnóstico sospechado de dengue, según los criterios de la OMS $(1,20)$ y la OPS (21), que acudieron al servicio de urgencias del Hospital General Dr. Bernardo J. Gastélum de Culiacán en el período señalado y que en sus historias clínicas constaran los resultados de los siguientes exámenes de laboratorio: concentración de hemoglobina, transaminasa glutámico-oxalacética (TGO), transaminasa glutámico-pirúvica (TGP) y albúmina sérica; hematocrito; conteo de leucocitos, neutrófilos y plaquetas; y tiempo de protrombina (TP) y tiempo parcial de tromboplastina (TPT).

En el estudio se incluyeron todos los casos de dengue hemorrágico positivos a anticuerpos específicos de la clase IgM contra el virus del dengue, según los cri- 
terios diagnósticos de la OMS (1). Este diagnóstico se realizó mediante la técnica de inmunoensayo enzimático de captura de anticuerpos (MAC-ELISA) con una mezcla de antígenos de los cuatro serotipos del virus del dengue, producido por el Departamento de Virología Diagnóstica del Instituto de Diagnóstico y Referencia Epidemiológicos (InDRE), de México, D.F. Los casos se confirmaron mediante el aislamiento viral por cultivo en células de mosquito con identificación por inmunofluorescencia directa, o mediante aislamiento e identificación del ARN viral en muestras clínicas mediante la técnica de transcripción inversa seguida de la reacción en cadena de la polimerasa (22). Las muestras se obtuvieron después del quinto día de la aparición de los síntomas de la enfermedad, se conservaron en refrigeración $\left(2-8^{\circ} \mathrm{C}\right) \mathrm{du}-$ rante un lapso no mayor de 48 horas y se enviaron al InDRE para su análisis.

Se estimó que para analizar con un adecuado nivel de significación ( $\alpha=0,05$ y $\beta=0,95)$ las diferencias de proporciones en la presentación de las manifestaciones hemorrágicas en los casos de dengue y de dengue hemorrágico se necesitaba un mínimo de 37 pacientes.

Para la captación de las variables en estudio se utilizó un cuestionario elaborado específicamente para identificar las características clínicas y epidemiológicas del dengue y el dengue hemorrágico.

\section{Análisis de los datos}

Para las variables cuantitativas se calcularon la media y la desviación estándar, mientras que para las variables cualitativas se calcularon las frecuencias absolutas y relativas de cada categoría, con sus respectivos intervalos de confianza de 95\% (IC95\%). Para comparar los grupos se utilizaron las pruebas de la ji al cuadrado y exacta de Fisher, y la diferencia entre medias se contrastó mediante la prueba de la $t$ de Student. Se calcularon las razones de posibilidades (odds ratio, OR) crudas y ajustadas para comparar las características clínicas y sociodemográficas entre los pacientes con dengue y dengue hemorrágico.

La asociación entre las variables independientes (las características clínicas y epidemiológicas de los pacientes) y la variable dependiente (diagnóstico confirmado de dengue hemorrágico) se realizó mediante el análisis de regresión simple. Las variables que mostraron una relación significativa $(P<0,05)$ se incluyeron en el análisis de regresión logística multifactorial.

El análisis de los datos se realizó mediante el paquete estadístico Stata v. 6.0.

\section{RESULTADOS}

En los dos meses de estudio se presentaron en el Hospital General de Culiacán 494 casos de dengue (435 de dengue y 59 de dengue hemorrágico), de los cuales solo 241 cumplieron los criterios de inclusión para este estudio (207 de dengue y 34 de dengue hemorrágico) (23). La edad promedio de los pacientes fue de 34,7 $\pm 15,1$ años (mínimo: 8; máximo: 86); $140(58,1 \%)$ eran mujeres o niñas y 101 $(41,9 \%)$ eran hombres o niños.

La relación entre los casos de dengue y de dengue hemorrágico fue aproximadamente de 6:1, con más personas del sexo masculino con dengue hemorrágico y más del sexo femenino con dengue $(P=0,001)$. La media de la edad fue similar en ambas formas de la enfermedad: $34,5 \pm 15,3$ años en los casos de dengue y $35,3 \pm 14,2$ años en los de dengue hemorrágico (cuadro 1); no se observaron casos de dengue hemorrágico en menores de 14 años de edad. Asimismo, la relación de casos de dengue y dengue hemorrágico fue similar en los diferentes grupos de edad.

Los antecedentes de dengue y los casos de esta enfermedad en la familia fueron similares en los grupos de pacientes con dengue y con dengue hemorrágico, sin embargo en el segundo se encontró un número significativamente mayor de casos de dengue en la comunidad que en el primero $(94,1 \%$ frente a $78,3 \%$, respectivamente; $P=0,031$ ) (cuadro 1).

El tiempo transcurrido entre la aparición de los síntomas y el inicio del tratamiento en la población estudiada fue de 3,5 \pm 2,2 días (mínimo: 0, máximo: 14), mayor en el grupo de pacientes con dengue hemorrágico que en el de dengue $(4,6 \pm 2,3$ frente a $3,3 \pm 2,2$, respectivamente; $P=0,023$ ) (cuadro 1 ).

Los síntomas generales se presentaron en similar proporción en ambos grupos, excepto por una mayor presencia de vómitos en el grupo con dengue hemorrágico $(67,7 \%)$ que en el de dengue $(47,3 \%$; $P=0,028)$. Como era de esperar, en el grupo de pacientes con dengue hemorrágico se presentaron muchos más casos con hemorragias espontáneas - gingivorragia $(P<0,001)$, epistaxis $(P=0,015)$, hematemesis $(P=0,030)$ y melena $(P<0,001)$ - así como con presencia de ascitis, derrame pleural, sangrado y hemoconcentración, que en el grupo con dengue $(P<0,001)$. No se encontraron diferencias significativas entre ambos grupos con respecto a la presencia de petequias, equimosis y resultados positivos a la prueba del torniquete $(P>0,05)$.

En los casos con dengue hemorrágico se observaron mayores niveles de hemoglobina, hematocrito $(P<0,001)$ y TGO $(P=0,020)$ que en los de dengue. Por el contrario, el grupo de pacientes con dengue hemorrágico presentó niveles significativamente menores de neutrófilos $(P=0,002)$, plaquetas $(P<0,001)$ y albú-

\section{CUADRO 1. Características epidemiológicas de los casos de dengue. Hospital General de Culia- cán, Sinaloa, México, 2003}

\begin{tabular}{|c|c|c|c|}
\hline Variable & Dengue (\%) & Dengue hemorrágico (\%) & $P$ \\
\hline Total de pacientes estudiado & 207 & 34 & \\
\hline \multicolumn{4}{|l|}{ Sexo } \\
\hline Hombre & $78(37,7)$ & $23(67,7)$ & $0,001^{a}$ \\
\hline Mujer & $129(62,3)$ & $11(32,4)$ & $0,001^{a}$ \\
\hline Edad (años \pm desviación estándar) & $34,5 \pm 15,3$ & $35,3 \pm 14,2$ & $0,823^{b}$ \\
\hline$<25$ & $59(28,5)$ & $9(26,5)$ & $0,806^{a}$ \\
\hline $25-34$ & $48(23,2)$ & $9(26,5)$ & $0,676^{a}$ \\
\hline $35-44$ & $35(16,9)$ & $7(20,6)$ & $0,600^{\mathrm{a}}$ \\
\hline $45-64$ & $39(18,8)$ & $7(20,6)$ & $0,810^{\mathrm{a}}$ \\
\hline$\geq 65$ & $13(6,3)$ & $1(2,9)$ & $0,440^{\circ}$ \\
\hline Infección previa de dengue & $46(22,2)$ & $6(17,7)$ & $0,539^{a}$ \\
\hline Casos en la familia & $95(45,9)$ & $10(29,4)$ & $0,072^{\mathrm{a}}$ \\
\hline Casos en la comunidad & $162(78,3)$ & $32(94,1)$ & $0,031^{a}$ \\
\hline \multicolumn{4}{|l|}{ Tiempo desde los primeros síntomas } \\
\hline días ( \pm desviación estándar) & $3,3 \pm 2,2$ & $4,6 \pm 2,3$ & $0,023^{b}$ \\
\hline
\end{tabular}

a Según las pruebas de la ji al cuadrado. Nivel de significación $P<0,05$.

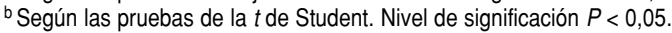

c Según la prueba exacta de Fisher. Nivel de significación $P<0,05$. 
CUADRO 2. Frecuencia de las características clínicas en los casos de dengue y dengue hemorrágicos. Hospital General de Culiacán, Sinaloa, México, 2003

\begin{tabular}{|c|c|c|c|}
\hline Característica clínica & Dengue (\%) & $\begin{array}{c}\text { Dengue } \\
\text { hemorrágico (\%) }\end{array}$ & $P^{a}$ \\
\hline \multicolumn{4}{|l|}{ Signos y síntomas generales } \\
\hline Fiebre & $199(96,1)$ & $34(100)$ & 0,244 \\
\hline Dolor de cabeza & $197(95,2)$ & $33(97,1)$ & 0,625 \\
\hline Mialgias & $195(94,2)$ & $33(97,1)$ & 0.494 \\
\hline Artralgias & $190(91,8)$ & $31(91,2)$ & 0,905 \\
\hline Dolor retrocular & $152(73,4)$ & $23(67,7)$ & 0,483 \\
\hline Exantema & $40(19,3)$ & $8(23,5)$ & 0,569 \\
\hline Conjuntivitis & $39(18,8)$ & $8(23,5)$ & 0,522 \\
\hline Náuseas & $149(72,0)$ & $29(85,3)$ & 0,102 \\
\hline Vómito & $98(47,3)$ & $23(67,7)$ & 0,028 \\
\hline Dolor abdominal & $51(24,6)$ & $13(38,2)$ & 0,096 \\
\hline \multicolumn{4}{|l|}{ Síntomas de mayor gravedad } \\
\hline Petequias & $17(8,2)$ & $4(11,8)$ & 0,496 \\
\hline Equimosis & $2(1,0)$ & $1(2,9)$ & 0,336 \\
\hline Prueba del torniquete positiva & $15(7,3)$ & $4(11,8)$ & 0,365 \\
\hline Gingivorragia & $13(6,3)$ & $12(35,3)$ & $<0,001$ \\
\hline Epistaxis & $12(5,8)$ & $6(17,7)$ & 0,015 \\
\hline Hematemesis & $7 \quad(3,4)$ & $4(11,8)$ & 0,030 \\
\hline Melena & $1(0,5)$ & $5(14,7)$ & $<0,001$ \\
\hline Trombocitopenia & $63(30,4)$ & $34(100)$ & $<0,001$ \\
\hline Presencia de ascitis & $4(1,9)$ & $10(29,4)$ & $<0,001$ \\
\hline Derrame pleural & $4 \quad(1,9)$ & $6(17,7)$ & $<0,001$ \\
\hline Sangrado & $52(25,1)$ & $30(88,2)$ & $<0,001$ \\
\hline Hemoconcentración & $12(5,8)$ & $20(58,8)$ & $<0,001$ \\
\hline \multicolumn{4}{|l|}{ Parámetros de laboratorio ( \pm desviación estándar) } \\
\hline Hemoglobina (g/dL) & $14,15 \pm 1,60$ & $16,14 \pm 2,20$ & $<0,001$ \\
\hline Hematocrito (\%) & $41,34 \pm 4,79$ & $47,24 \pm 6,25$ & $<0,001$ \\
\hline Leucocitos $\left(/ \mathrm{mm}^{3}\right)$ & $4774 \pm 2008$ & $4231 \pm 1706$ & 0,250 \\
\hline Neutrófilos (/mm³) & $3050 \pm 1590$ & $1905 \pm 974,6$ & 0,002 \\
\hline Plaquetas $\left(1000 / \mathrm{mm}^{3}\right)$ & $147,62 \pm 91,98$ & $31,04 \pm 19,34$ & $<0,001$ \\
\hline Transaminasa glutámico-oxalacética (Ul/dL) & $77,83 \pm 101,44$ & $151,33 \pm 142,25$ & 0,020 \\
\hline Transaminasa glutámico-pirúvica (UI/dL) & $65,84 \pm 86,17$ & $106,5 \pm 99,35$ & 0,118 \\
\hline Albúmina (g/dL) & $3,8 \pm 0,5$ & $3,1 \pm 0,4$ & $<0,001$ \\
\hline Tiempo de protrombina (s) & $12,7 \pm 1,3$ & $12,5 \pm 0,9$ & 0,657 \\
\hline Tiempo parcial de protrombina (s) & $37,2 \pm 5,6$ & $41,1 \pm 6,47$ & 0,030 \\
\hline
\end{tabular}

a Según la prueba de la ji al cuadrado. Se aplicó la prueba exacta de Fisher cuando los valores observados en la tabla de contingencia fueron menores de 5 . Nivel de significación $P<0,05$.

${ }^{b}$ En 3 casos clasificados como dengue hemorrágico no se manifestaron hemorragias francas, solo hiperemia conjuntival.

mina $(P=0,001)$ que el de dengue (cuadro 2). Cabe señalar que, de acuerdo con los parámetros establecidos por la OMS, todos los casos clasificados como dengue hemorrágico presentaron trombocitopenia (menos de 100000 plaquetas $/ \mathrm{mm}^{3}$ ), aunque solo $32,6 \%$ de los casos de dengue tenían esta condición $(P<0,001)$.
Algunos casos que tenían los valores plaquetarios más bajos también presentaron equimosis y petequias, pero al no cumplir con todos los criterios de clasificación de dengue hemorrágico establecidos por la OMS, se consideraron como casos de dengue; un caso de dengue hemorrágico con conteo de plaquetas de $8690 / \mathrm{mm}^{3}$ presentó gingivorragia y petequias.

Los resultados del análisis de regresión simple se presentan en el cuadro 3. Como se puede observar, un conteo plaquetario inferior a $20000 / \mathrm{mm}^{3}$ está asociado con las siguientes manifestaciones hemorrágicas de la enfermedad $(P<0,05)$ : epistaxis $(\mathrm{OR}=5,08$; IC95\%: $1,71$ a 14,97$)$, petequias $(\mathrm{OR}=4,00$; IC95\%: 1,39 a 11,45) y gingivorragia $(\mathrm{OR}=3,09$; IC95\%: 1,10 a 8,63). Cuando el número de plaquetas se encontraba entre $20001 / \mathrm{mm}^{3}$ y $40000 / \mathrm{mm}^{3}$ se mantuvo la asociación significativa $(P<0,05)$ con la presencia de petequias $(\mathrm{OR}=3,60$; IC95\%: 1,26 a 10,22) y gingivorragia $(\mathrm{OR}=2,78$; IC95\%: 1,01 a 7,70). El número de plaquetas superior a $100000 / \mathrm{mm}^{3}$ resultó un factor significativo de protección $(P<0,05)$ contra la presencia de petequias $(\mathrm{OR}=0,30$; IC95\%: $0,15$ a 0,95$)$ y gingivorragia $(\mathrm{OR}=0,14$; IC95\%: 0,05 a 0,39).

Algunos de los más notorios signos de gravedad, como la presencia de ascitis, hematemesis, gingivorragia, vómitos persistentes, plaquetopenia e hiperemia conjuntival, se asociaron con una mayor probabilidad de diagnóstico de dengue hemorrágico (cuadro 3).

Según los resultados del análisis multifactorial ajustado por la edad, el sexo y la presencia de casos de dengue en la localidad, las variables con valor predictivo de una mayor gravedad de la enfermedad fueron: la presencia de ascitis $(\mathrm{OR}=22,12$; IC95\%: 5,00 a 97,87), la gingivorragia (OR = 7,35; IC95\%: 2,11 a 25,61 ), la hematemesis (OR = 7,40; IC95\%: 1,04 a 52,42 ), la trombocitopenia (plaquetas entre $40001 / \mathrm{mm}^{3}$ y $60000 / \mathrm{mm}^{3}$ ) $(\mathrm{OR}=5,43$; IC95\%: 1,58 a 18,72$)$, la hiperemia conjuntival $(\mathrm{OR}=4,27$; IC $95 \%$ : 1,37 a 13,28), los vómitos persistentes $(\mathrm{OR}=3,04$; IC95\%: 1,05 a 8,80) y la ausencia de congestión nasal $(\mathrm{OR}=0,015$ IC95\%: 0,0004 a 0,473) (cuadro 4).

CUADRO 3. Asociación entre el número de plaquetas y las variables hemorrágicas. Hospital General de Culiacán, Sinaloa, México, 2003

\begin{tabular}{|c|c|c|c|c|c|c|}
\hline \multirow[b]{3}{*}{ Síntoma hemorrágico } & \multicolumn{6}{|c|}{ Conteo de plaquetas } \\
\hline & $<20000$ & $20001-40000$ & $40001-60000$ & $60001-80000$ & $80001-100000$ & $>100000$ \\
\hline & OR $(\operatorname{IC} 95 \%)^{a}$ & OR (IC95\%) & OR (IC95\%) & OR (IC95\%) & OR (IC95\%) & OR (IC95\%) \\
\hline Epistaxis & $5,08(1,71-14,97)$ & $4,27 \quad(0,05-3,33)$ & $1,34(0,28-6,28)$ & $1,25(0,15-10,37)$ & $N A^{b}$ & $0,51(0,19-1,34)$ \\
\hline Petequias & $4,00(1,39-11,45)$ & $3,60(1,26-10,22)$ & NA & NA & $1,05(0,12-8,62)$ & $0,30(0,15-0,95)$ \\
\hline Gingivorragia & $3,09(1,10-8,63)$ & $2,78 \quad(1,01-7,70)$ & $2,22(0,68-7,24)$ & $(0,40-9,8)$ & $2,00 \quad(0,40-9,8)$ & $0,14(0,05-0,39)$ \\
\hline
\end{tabular}

a OR: razón de posibilidades (odds ratio); IC95\%: intervalo de confianza de 95\%.

b NA: no applicable por no haber pacientes en esta categoría. 
CUADRO 4. Síntomas asociados ${ }^{a}$ con los casos de dengue hemorrágico. Hospital General de Culiacán, Sinaloa, México, 2003

\begin{tabular}{lccrr}
\hline \multicolumn{1}{c}{ Síntoma } & \multicolumn{2}{c}{ No. de casos (\%) } & & \multirow{2}{*}{ IC95\% $^{\text {c }}$} \\
\cline { 2 - 3 } & Con el síntoma & Sin el síntoma & OR & $2,11-25,61$ \\
Gingivorragia & $12(35,3)$ & $22(64,7)$ & 7,35 & $1,05-8,80$ \\
Vómitos persistentes & $15(44,1)$ & $19(55,9)$ & 3,04 & $5,00-97,87$ \\
Presencia de ascitis & $10(29,4)$ & $24(70,6)$ & 22,12 & $1,58-18,72$ \\
Trombocitopeniad & $8(23,5)$ & $26(76,5)$ & 5,43 & $0,0004-0,473$ \\
Ausencia de congestión nasal & $1(2,9)$ & $33(97,1)$ & 0,015 & $1,37-13,28$ \\
Hiperemia conjuntival & $8(23,5)$ & $26(76,5)$ & 4,27 & $1,04-52,42$ \\
Hematemesis & $4(11,8)$ & $30(88,2)$ & 7,40 &
\end{tabular}

a Análisis multifactorial ajustado por la edad, el sexo y la presencia de casos de dengue en la localidad.

b OR: razón de posibilidades (odds ratio).

c IC95\%: intervalo de confianza de 95\%.

${ }^{d}$ La trombocitopenia se definió como un conteo plaquetario entre 40001 y $60000 / \mathrm{mm}^{3}$.

\section{DISCUSION}

En la muestra estudiada, el diagnóstico de dengue hemorrágico fue más frecuente en los pacientes que informaron de la presencia de casos de dengue en su comunidad, aunque no se observó una asociación con el diagnóstico previo de dengue o dengue hemorrágico. Esto podría estar en contradicción con lo informado por Guzmán y colaboradores (24) en el sentido de que la respuesta inmunitaria humoral a una infección primaria por un determinado serotipo origina anticuerpos neutralizantes capaces de proteger al individuo contra el virus homólogo $\mathrm{y}$, en menor medida, contra otros serotipos. Por otra parte, este efecto subneutralizante de los anticuerpos generados por infecciones heterólogas previas está asociado con algunos procesos inmunopatogénicos que llevan a las formas graves de la enfermedad mediante el llamado efecto de amplificación dependiente de anticuerpos (24).

Otros autores consideran que la combinación de algunos elementos del medio ambiente y ciertos mecanismos inmunológicos podrían llevar a que los patrones epidemiológicos del dengue estén en consonancia con un período de corta duración de inmunidad cruzada que no depende de la heterogeneidad de las infecciones previas o del efecto de amplificación dependiente de anticuerpos (25-27).

Otra posible explicación podría estar relacionada con la virulencia de las cepas causantes de la enfermedad grave, que podría influir en la relación entre el número de casos graves y el número total de personas infectadas. Hasta el momento no se ha aislado la variante americana del genotipo viral DENV-2 a partir de casos de dengue hemorrágico. Según estudios de laboratorio, este genotipo tiene una menor virulencia que su variante asiática, que se transmite más fácilmente por los mosquitos vectores y se ha asociado con brotes de dengue hemorrágico $(28,29)$.

La mayor frecuencia de hipoalbuminemia y hemoconcentración encontrada en los casos de dengue hemorrágico son de importancia clínica, ya que ambas condiciones pueden provocar hipotensión arterial y choque por dengue. En el presente estudio se encontró una fuerte asociación entre la fuga capilar (presencia de ascitis) y el diagnóstico de dengue hemorrágico, similar a lo observado por otros investigadores $(30,31)$. Aunque algunos autores refieren que la administración temprana de fluidos intravenosos puede enmascarar la presencia de la hemoconcentración provocada por una excesiva pérdida de sangre debida a hemorragias (32), en la muestra estudiada se encontró una mayor frecuencia de hemoconcentración y otros indicadores de hemorragia en los casos de dengue hemorrágico que en los casos de dengue $(P<0,001)$. Esta diferencia podría deberse a que por dificultades para clasificar oportunamente los casos de dengue hemorrágico, no se aplicara a tiempo el tratamiento con fluidos intravenosos que evitan la hemoconcentración.

Según Rigau y colaboradores no es fácil detectar los casos graves de dengue mediante criterios simples. Si bien la definición ideal de caso de dengue hemorrágico debería ser sencilla, simple y suficientemente explícita para guiar a los médicos y los epidemiólogos en la identificación de los casos a partir del examen físico y los datos de laboratorio recogidos en los registros hospitalarios, esto no siempre es posible. Por ello se ha propuesto como medida de vigilancia no invasiva la medición estricta y frecuente de la tensión arterial como indicador de la posible evolución de la enfermedad a formas más graves $(33,34)$.

La casi universalidad de los síntomas del dengue hemorrágico se confirma al comparar los resultados obtenidos en este estudio con los encontrados en otras regiones del mundo. Rahman y colaboradores (35) encontraron en los pacientes de un hospital de Dhaka, Bangladesh, que los síntomas característicos de dengue hemorrágico fueron fiebre $(100 \%)$, dolor de cabeza $(91 \%)$, mialgia $(85 \%)$ y artralgia (85\%), cifras similares a las encontradas en los casos de dengue hemorrágico estudiados aquí (100\%, 97,1\%, $97,1 \%$ y $91,2 \%$, respectivamente). Llama la atención que a pesar de la similitud en los síntomas, la mayoría de los pacientes bengalíes $(80 \%)$ se presentara al hospital después de 5 días de fiebre, dos días más tarde que el promedio de los pacientes del presente estudio (3,5 $\pm 2,2$ días). Esto puede atribuirse a diferencias en las niveles de conocimientos de estas poblaciones acerca del dengue y de acceso a los servicios de salud.

También hay similitudes con un estudio de Krishnamurti y colaboradores (36) en el sentido de que se encontraron casos con coagulopatía, trombocitopenia $\mathrm{y}$ hemorragias francas, tanto en los pacientes con dengue como con dengue hemorrágico.

En otro estudio realizado en la India para validar el valor predictivo de los perfiles alterados del tiempo de protrombina, el tiempo parcial de protrombina y la trombocitopenia $(<50$ 000) en casos de dengue hemorrágico, se encontraron tiempos de protrombina alterados únicamente en los casos con hemorragias espontáneas (37). En el presente estudio se observaron valores normales en ambos grupos (con dengue y con dengue hemorrágico), aunque se debe señalar que si bien el tiempo parcial de protrombina presentó valores dentro de los límites normales en ambos grupos, en los casos de dengue hemorrágico fue significativamente mayor $(P=0,03)$.

La concentración de marcadores de la coagulación tiene importancia clínica, ya que puede indicar una mayor o menor actividad fibrinolítica. Se ha observado que la reducción en la concentración de fibrinógeno en presencia de trombocitopenia se asocia significativamente con la presencia de hemorragias intensas (36). 
Además, Halstead y colaboradores han encontrado anticuerpos de reacción cruzada contra el plasminógeno durante y después de la infección con dengue (38), mientras otros autores sugieren que el aumento de la actividad fibrinolítica podría indicar una interacción directa del virus del dengue con el plasminógeno. Otra posible explicación podría ser que durante la infección se libera heparán o sulfato de condroitina a partir del glucocáliz, moléculas similares a la heparina que pueden dificultar la coagulación (39).

Se confirmó que el resultado de la prueba del torniquete es uno de los marcadores diagnósticos menos sensibles de dengue hemorrágico (40) y que no hay diferencias significativas en cuanto a la presencia de manifestaciones hemorrágicas en pacientes con trombocitopenia y sin ella $(33,41)$. Esto podría indicar que no se debe sobrevalorar la utilidad de la presencia de manifestaciones hemorrágicas y de los conteos plaquetarios como factores independientes de predicción de la evolución hacia formas graves de la enfermedad.

No obstante, las asociaciones mostradas por el análisis de regresión simple entre el conteo plaquetario inferior a $20000 / \mathrm{mm}^{3}$ y la presencia de epistaxis, petequias y gingivorragia, por una parte, y entre el conteo de $20001 / \mathrm{mm}^{3}$ a $40000 / \mathrm{mm}^{3}$ y la presencia de petequias y gingivorragia, por otra, podrían respaldar la utilidad de este indicador como criterio para la hospitalización y seguimiento de los pacientes, ya que un descenso progresivo en el número de plaquetas puede anticipar la evolución a formas graves de dengue (32). Por otro lado, un número de plaquetas superior a $100000 / \mathrm{mm}^{3}$ podría indicar una evolución benigna de la enfermedad, al menos en cuanto a las manifestaciones hemorrágicas (42).

La asociación entre la presencia de ascitis, gingivorragia, hematemesis, trombocitopenia (con valores de plaquetas entre $40001 / \mathrm{mm}^{3}$ y $60000 / \mathrm{mm}^{3}$ ) y vómitos persistentes, por un lado, y el diagnóstico de dengue hemorrágico, por el otro, confirma su valor como signos de alarma que anuncian la inminencia del choque por dengue $(21,43)$.

El hecho de no haber observado conteos plaquetarios inferiores a $100000 / \mathrm{mm}^{3}$ - parámetro establecido por la OMS como necesario para clasificar los casos como dengue hemorrágico- en varios casos de dengue que presentaban características clínicas compatibles con el dengue hemorrágico impidió que se cla- sificaran como tales al menos cinco casos con fuga capilar. Otros investigadores $(32,42,44)$ también han manisfestado su discrepancia con la clasificación de la OMS de dengue hemorrágico/síndrome de choque por dengue debido, entre otros argumentos, a que la definición operativa de caso de dengue hemorrágico establece que el paciente debe mostrar fuga plasmática, hemorragias espontáneas, trombocitopenia y hemoconcentración. Sin embargo, se debe tener en cuenta la secuencia de estos eventos, ya que estos parámetros pueden estar o no presentes simultáneamente (45).

Teniendo como fondo las dificultades ya mencionadas para la clasificación de los casos de dengue por la rigidez de los parámetros establecidos por la OMS, en el marco del II Congreso Internacional de Dengue y Fiebre Amarilla, celebrado en La Habana en mayo de 2004, investigadores de 42 países compartieron sus experiencias y se llegó a una visión más amplia de esta enfermedad. Esta experiencia contribuyó a enriquecer los criterios de diagnóstico clínico utilizados en el presente estudio y respaldó la utilidad de realizar pruebas serológicas a todos los casos sospechados para lograr una mayor precisión en el diagnóstico, sobre todo en los casos en los que se requería un diagnóstico diferencial.

$\mathrm{Al}$ analizar los resultados del presente estudio se deben tener en cuenta algunas limitaciones. En primer lugar, la falta de confirmación mediante el aislamiento viral de todos los casos de dengue - y no solo de los casos de dengue hemorrágico- y la no repetición de las pruebas de laboratorio en muchos casos de dengue hemorrágico impidieron observar las variaciones en los niveles de plaquetas, hematocrito, hemoglobina y otros resultados de laboratorio. La OMS propone observar los casos de dengue cada una hora para poder detectar la disminución en el número de plaquetas y la elevación del hematocrito, como criterios esenciales de diagnóstico temprano de dengue hemorrágico (1). Tampoco se solicitaron pruebas de ultrasonido a los pacientes con dengue hemorrágico ni se documentó la presencia de enfermedades crónicas en los pacientes estudiados. Además, no se buscó la presencia de anticuerpos de la clase IgG contra el virus del dengue en los pacientes, por lo que no se pudo relacionar con suficiente certeza si el haber padecido dengue previamente fue una condición de riesgo de dengue hemorrágico. Debido al carácter transversal de este estudio no fue posible medir la incidencia.

A pesar de estas limitaciones, los resultados de este estudio han permitido: a) demostrar la presencia masiva de casos de dengue en el Estado de Sinaloa, México, algo que se ha negado y que ha dificultado la referencia y el tratamiento oportunos de los pacientes; b) caracterizar la enfermedad y las manifestaciones clínicas del dengue y el dengue hemorrágico en los casos diagnosticados en esta entidad federativa; c) adquirir experiencia directa en el diagnóstico específico de los casos de dengue hemorrágico. Solo uno de los pacientes estudiados evolucionó a dengue hemorrágico/síndrome de choque por dengue, pero logró recuperarse y no se observaron manifestaciones clínicas atípicas, como fallo hepático fulminante, miocardiopatía o trastornos neurológicos. Durante el período de estudio y hasta la fecha de este informe no se ha presentado ninguna defunción por dengue en el Hospital General de Culiacán Dr. Bernardo J. Gastélum.

El incremento sostenido en el número de casos de dengue y dengue hemorrágico en el Estado de Sinaloa obliga a mantener la capacitación y la actualización constantes del personal médico y paramédico, especialmente en cuanto a la fisiopatología del dengue hemorrágico, para evitar muertes prevenibles. Asimismo, se deben llevar a cabo campañas permanentes de difusión dirigidas a la población para que conozca no solo los aspectos relacionados con el control del vector, sino también los síntomas del dengue y el dengue hemorrágico, con lo que se propicia la solicitud oportuna de atención médica.

Agradecimientos. Se agradece a Maura Sara Castañeda, coordinadora de la Maestría en Salud Pública con área de concentración en Epidemiología, ya que el presente artículo es una síntesis del trabajo de tesis de maestría en Salud Pública con área de concentración en Epidemiología de la primera autora. Asimismo, se agradece a Javier Mota Sánchez por su asesoría; a Humberto Soto Valenzuela, jefe de Vigilancia Epidemiológica y al personal médico y de enfermería del Hospital General de Culiacán, Sinaloa, México, y a las estudiantes de la Facultad de Medicina de la Universidad Autónoma de Sinaloa Norma Leticia García Sañudo y Alondra Alejandra Cárdenas Angulo por su valioso apoyo. 


\section{REFERENCIAS}

1, World Health Organization. Dengue haemorrhagic fever: diagnosis, treatment, prevention and control. 2nd ed. Geneva: World Health Organization; 1997.

2. Pan American Health Organization. 44th Directing Council. Resolution CD44.R9. Dengue. Washington, D.C.: PAHO; 2003. Hallado en http://www.paho.org/english/gov/cd/ cd44-r9-e.pdf. Acceso el 30 de abril de 2008.

3. World Health Organization. Dengue and dengue haemorrhagic fever. Geneva: WHO; 2008. Hallado en http://www.who.int/mediacentre/ factsheets/fs117/en/. Acceso el 2 de diciembre de 2008 .

4. Calisher $\mathrm{CH}$. Persistent emergence of dengue. Emerg Infect Dis. 2005;11:738-9.

5. Gubler DJ. The changing epidemiology of yellow fever and dengue, 1900 to 2003: full circle? Comp Immunol Microbiol Infect Dis. 2004;27:319-30.

6. Guzmán MG, Kourí G. Dengue: an update. Lancet Infect Dis. 2002;2:33-42.

7. Hopp MJ, Foley JA. Worldwide fluctuations in dengue fever cases related to climate variability. Climate Res. 2003;25:85-94.

8. Depradine CA, Lovell EH. Climatological variables and the incidence of dengue fever in Barbados. Int J Environ Health Res. 2004;14: 429-41.

9. Guang W, Qing W, Ono M. Investigation on Aedes aegypti and Aedes albopictus in the northwestern part of Hainan Province. China Trop Med. 2005;5:230-3.

10. Guzmán MG, Kourí G. Dengue and dengue hemorrhagic fever in the Americas: lessons and challenges. J Clin Virol. 2003;27:1-13.

11. Mackenzie JS, Gubler DJ, Petersen LT. Emerging flaviviruses: the spread and resurgence of Japanese encephalitis, West Nile and dengue viruses. Nature Med. 2004;10:S98-A109.

12. Meltzer MI, Riga JG, Clark GG, Reiter P, Gubler DJ. Using disability adjusted life years to assess the economic impact of dengue in Puerto Rico: 1984-1994. Am J Trop Med Hyg. 1998;59:265-71.

13. Gubler DJ. Epidemic dengue/dengue hemorrhagic fever as a public health, social and economic problem in the 21st century. Trends Microbiol. 2002;10(1):100-3.

14. Organización Panamericana de la Salud. Salud en las Américas 2007. Vol. I. Washington, D.C.: OPS; 2007. (Publicación Científica y Técnica No. 622.)

15. Pan American Health Organization. Reported cases of dengue and dengue hemorrhagic fever (DHF), Region of the Americas (by country and subregion). Figures for 2007 (to week noted by each country). Washington, D.C.: PAHO; 2008. Hallado en http://www. paho.org/english/AD/DPC/CD/denguecases-2007.htm. Acceso el 26 de noviembre de 2008.

16. Gómez-Dantés H, Rodríguez-López $\mathrm{MH}$. El dengue y la fiebre hemorrágica del dengue: clínica y epidemiología. México, D.F.: Asociación Mexicana de Infectología y Microbiología Clínica; 2000.

17. Estados Unidos Mexicanos, Secretaría de Salud. Anuarios de mortalidad 1984-2007. México, D.F.: Secretaría de Salud; 2008. Ha- llado en http://www.dgepi.salud.gob.mx/ anuario/index.html\#. Acceso el 26 de noviembre de 2008

18. Estados Unidos Mexicanos, Secretaría de Salud. Indicadores básicos de Salud, 20002004. Indicadores de morbilidad. México, D.F.: Secretaría de Salud; 2005. Hallado en http://www.mex.ops-oms.org/documentos/ indicadores/excel/ibs morbi2000-2004.xls. Acceso el 26 de noviembre de 2008.

19. Dirección General de Epidemiología. Manual simplificado para la vigilancia epidemiológica del dengue. México, D.F.: Dirección General de Epidemiología; 1997. Hallado en http://www.dgepi.salud.gob.mx/infoepi/ manuales/Man19-Dengue/Man19.htm. Acceso el 2 de diciembre de 2008.

20. World Health Organization. Guidelines for treatment of dengue fever/dengue hemorrhagic fever in small hospitals. New Delhi: WHO Regional Office for South-East Asia; 1999.

21. Organización Panamericana de la Salud. Dengue y dengue hemorrágico. Guías para su prevención y control. Washington, D.C.: OPS 1995. (Publicación Científica No. 548.)

22. Secretaría de Salud, Instituto Nacional de Referencia Epidemiológica. Manual de procedimientos de laboratorio para dengue. México, D.F.: Instituto Nacional de Referencia Epidemiológica; 1997

23. Hospital General de Culiacán. Informe anual estadistico del año 2003 del Hospital General de Culiacán Dr. Bernardo J. Gastelum. Culiacán: Servicios de Salud de Sinaloa; 2008. Hallado en http://hgculiacan.com/estadisticas/ estadisticas.htm. Acceso el 26 de noviembre de 2008.

24. Guzmán MG, García G, Kourí G. Dengue y fiebre hemorrágica del dengue: un problema de salud mundial. Rev Cubana Med Trop. 2008;60(1): sin páginas. Hallado en http:/ / bvs. sld.cu/revistas/mtr/vol60 1 08/mtr01108. $\mathrm{htm}$. Acceso el 26 de noviembre de 2008.

25. Kochel TJ, Watts DM, Halstead SB, Hayes CG Espinoza A, Felices V, et al. Effect of dengue-1 antibodies on American dengue-2 viral infection and dengue haemorrhagic fever. Lancet. 2002;360:310-2.

26. Adams B, Holmes EC, Zhang C, Mammen MP, Nimmannitya S, Kalayanarooj $S$, et al. Cross-protective immunity can account for the alternating epidemic pattern of dengue virus serotypes circulating in Bangkok. Proc Natl Acad Sci USA. 2006;103:14234-9.

27. Wearing HJ, Rohani P. Ecological and immunological determinants of dengue epidemics. Proc Natl Acad Sci USA. 2006;103:11802-7.

28. Rico-Hesse R. Microevolution and virulence of dengue viruses. Adv Virus Res. 2003;59. 315-41.

29. Cologna R, Armstrong PM, Rico-Hesse R. Selection for virulent dengue viruses occurs in humans and mosquitoes. J Virol. 2005;79: 853-9.

30. Rigau-Pérez JG. Clinical manifestations of dengue hemorrhagic fever in Puerto Rico, 1990-1991. Rev Panam Salud Publica. 1997 1(5):381-8.

31. Srikiatkhachorn A, Kruatrachue A, Ratanaprakarn W, Wongtapradit L, Nithipanya N Kalayanarooj S, et al. Natural history of plasma leakage in dengue hemorrhagic fever: a serial ultrasonographic study. Pediatr Infect Dis J. 2007;26:283-90.

32. Harris E, Videa E, Pérez L, Sandoval E, Téllez Y, Pérez ML, et al. Clinical, epidemiologic, and virologic features of dengue in the 1998 epidemic in Nicaragua. Am J Trop Med Hyg. 2000;63(1, 2):5-11.

33. Rigau-Pérez JG, Clark GG. Cómo responder a una epidemia de dengue: visión global y experiencia en Puerto Rico. Rev Panam Salud Publica. 2005;17(4):282-93.

34. Rigau-Pérez JG. Severe dengue: the need for new case definitions. Lancet Infect Dis. 2006;6 297-302.

35. Rahman M, Rahman K, Siddque A, Shoma S, Kamal A, Ali K, et al. First outbreak of dengue hemorrhagic fever, Bangladesh. Emerg Infect Dis. 2002;8(7):738-40.

36. Krishnamurti C, Kalayanarooj S, Cutting MA Peat RA, Rothwell SW, Reid TJ, et al. Mechanisms of hemorrhage in dengue without circulatory collapse. Am J Trop Med Hyg. 2001; 65:840-4.

37. Shivbalan S, Anandnathan K, Balasubramanian S, Datta M, Amairaj E. Predictors of spontaneous bleeding in Dengue. Indian J Pediatr. 2004;71(1):33-6.

38. Halstead SB. Dengue. Lancet. 2007;370: 1644-52.

39. Wills BA, Oragui EE, Dung NM, Loan HT, Chau NV, Farrar JJ, et al. Size and charge characteristics of the protein leak in dengue shock syndrome. J Infect Dis. 2004;190:810-8.

40. Gomber S, Ramachandran V, Kumar S, Agarwal KN, Gupta P, Gupta P, et al. Hematological observations as diagnostic markers in dengue hemorrhagic fever: a reappraisal. Indian J Pediatr. 2001;38:477-81.

41. Wakimoto MD, Azevedo MB, Sant' Anna de Oliveira J, Vommaro RV, Dornas JE. A experiência de um hospital público na vigilância e assistência aos casos de dengue durante a epidemia de 2002. Saúde em Foco/Informe Epidemiol Saúde Colectiva (Rio de Janeiro). 2002;24:25-62.

42. Narayanan M, Aravind MA, Ambikapathy $P$ Prema R, Jeyapaul MP. Dengue fever: clinical and laboratory parameters associated with complications. Dengue Bull. 2003;27:108-15.

43. Martínez Torres E. La prevención de la mortalidad por dengue: un espacio y un reto para la atención primaria de salud. Rev Panam Salud Publica. 2006;20(1):60-74.

44. Bandyopadhyay S, Lum LCS, Kroeger A. Classifying dengue: a review of the difficulties in using the WHO case classification for dengue hemorrhagic fever. Trop Med Internat Health. 2006;2(8):1238-55.

45. Navarrete-Espinosa J, Gómez-Dantés H, Celis-Quintal JG, Vázquez-Martínez JL. Clinical profile of dengue hemorrhagic fever cases in Mexico. Salud Publica Mex. 2005:47(3): 193-200.

Manuscrito recibido el 20 de febrero de 2008. Aceptado para publicación, tras revisión, el 30 de junio de 2008. 
ABSTRACT Objectives. To document the clinical and epidemiological characteristics present in dengue patients served by a hospital in Culiacan, Sinaloa, Mexico.

\section{Clinical and epidemiologic characteristics of dengue cases: the experience of a general hospital in Culiacan, Sinaloa, Mexico}

Methods. A cross-sectional, observational, and analytical study was carried out at the Hospital General Dr. Bernardo J. Gastélum de Culiacan from 1 October to 2 December 2003. Associations between the independent variables (the patients' clinical and epidemiological characteristics) and the dependent variable (confirmed hemorrhagic dengue) were determined through simple regression analysis. The variables that were significantly associated $(P<0.05)$ were submitted to multifactorial logistic regression analysis.

Results. Of the only 241 cases that met the study's inclusion criteria (207 dengue and 34 hemorrhagic dengue), the mean age was $34.7 \pm 15.1$ years. According to the results of the multifactorial analysis (adjusted by age, sex, and the presence of dengue cases at the geographic location), the variables predictive of major complications of the disease were: the presence of ascites (odds ration $[\mathrm{OR}]=22.12 ; 95 \%$ confidence interval [95\%CI]: 5.00-97.87), gingivorrhagia ( $\mathrm{OR}=7.35$; 95\%CI: 2.11-25.61), hematemesis $\left(\mathrm{OR}=7.40\right.$; 95\%CI: 1.04-52.42), thrombocytopenia (platelets from $40001 / \mathrm{mm}^{3}-$ $\left.60000 / \mathrm{mm}^{3}\right)(\mathrm{OR}=5.43 ; 95 \% \mathrm{CI}: 1.58-18.72)$, conjunctival hyperemia $(\mathrm{OR}=4.27$; 95\%CI: 1.37-13.28), persistent vomiting (OR $=3.04 ; 95 \% \mathrm{CI}: 1.05-8.80)$, and the absence of nasal congestion (OR $=0.015 ; 95 \% \mathrm{CI}$ : 0.0004-0.473).

Conclusions. The presence of ascites, gingivorrhagia, hematemesis, thrombocytopenia (with platelet values from $40001 / \mathrm{mm}^{3}-60000 / \mathrm{mm}^{3}$ ), and persistent vomiting were confirmed as warning signs of an imminent dengue attack. Platelet counts of $>100000 / \mathrm{mm}^{3}$ were confirmed in cases with acute clinical symptoms (capillary leak) that were not classified as hemorrhagic dengue due to falling short of the criteria established by WHO.

Key words Dengue, dengue hemorrhagic fever, signs and symptoms, epidemiology, Mexico. 